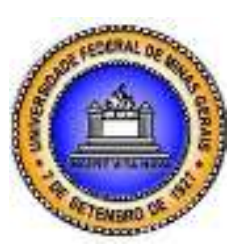

\title{
CÁLCULO DOS CUSTOS DOS PROCEDIMENTOS MÉDICOS HOSPITALARES EM HOSPITAIS BRASILEIROS ${ }^{1}$
}

\author{
COST CALCULUS OF HOSPITAL MEDICAL PROCEDURES IN BRAZILIAN \\ HOSPITALS
}

\section{CÁLCULO DE LOS COSTOS DE LOS PROCEDIMIENTOS HOSPITALARIOS EN HOSPITALES BRASILEÑOS}

Katia Abbas Correio

Universidade Estadual de Maringá

Email: katia_abbas@yahoo.com.br

\author{
Maury Leoncine \\ Planis Consultoria \\ Email: maury_leoncine@yahoo.com.br
}

\begin{abstract}
Resumo
O contexto do setor saúde no Brasil torna a gestão de custos uma ferramenta imprescindível. Para negociar um procedimento médico hospitalar, um hospital precisa saber quanto the custa este procedimento. Assim, o objetivo deste artigo é apresentar o cálculo dos custos do procedimento médico hospitalar parto cesárea em seis hospitais brasileiros que utilizam o método de custeio das seções homogêneas. O custo de um procedimento é obtido através do somatório dos custos dos centros produtivos, obtido através do método de custeio das seções homogêneas mais os custos dos materiais e medicamentos. As variações nos dados apurados nos hospitais pesquisados se devem ao perfil de atendimento, a taxa de ocupação, a ociosidade, muitas vezes elevada, ao valor da compra dos materiais e medicamento e, principalmente, ao custo com pessoal. A determinação do custo por procedimento é útil para estabelecer o preço médio de cada procedimento médico; estimar os custos de negociação de pacotes; determinar a margem bruta dos procedimentos médicos hospitalares e as margens brutas associadas às especialidades médicas; estimar a rentabilidade dos procedimentos médico hospitalares e das especialidades médicas; e confrontar com o valor faturado do custo do procedimento médico hospitalar.
\end{abstract}

Palavras chave: Hospitais, Método das seções homogêneas, Custo por procedimentos.

\begin{abstract}
The context of health sector in Brazil makes the cost management an indispensable tool. To negotiate a medical hospital, a hospital needs to know how much it costs this procedure. Thus, the aim of this paper is to present the costing of hospital medical procedure cesarean section in six Brazilian hospitals that use the cost method of homogeneous sections. The cost of a procedure is obtained by summing the costs of production centers, obtained by the method of costing more homogeneous sections of the costs of materials and medicines. The variations in data collected in the hospitals surveyed are due to the service profile, the occupancy rate, idleness, often high, the value of the purchase of materials and medicine, and especially the staff costs. Determining the cost per procedure is useful for establishing the average price of each medical procedure, to estimate the costs of negotiating packages and determine the gross margin of hospital and medical procedures gross margins associated with the medical specialties, to estimate the profitability of the hospital medical procedures and medical specialties, and confront the invoiced amount of the cost of hospital medical procedure.
\end{abstract}

Key-words: Hospitals, Method of homogeneous sections, cost per procedure.

\footnotetext{
${ }^{1}$ Submetido em 02 de agosto de 2012. Aceito em 02 de outubro de 2013. O artigo foi avaliado segundo o processo de duplo anonimato além de ser avaliado pelo editor. Editores responsáveis: Márcio Augusto Gonçalves e Lucas Maia dos Santos. Reprodução parcial ou total e trabalhos derivativos permitidos com a citação apropriada da fonte.
} 


\section{Resumem}

El contexto del sector de la salud en Brasil hace que la gestión de costes en una herramienta necesaria . Negociar un procedimiento médico del hospital, el hospital tiene que saber cuánto cuesta este procedimiento. El objetivo de este trabajo es presentar el cálculo de los costos de procedimiento médico hospitalario cesárea en seis hospitales brasileños utilizando el método del coste de las secciones homogéneas. El costo de un procedimiento se obtiene mediante la suma de los costos de los centros de producción, obtenidos por el método de financiación de las secciones homogéneas, más el costo de los materiales y medicamentos. Las variaciones en los datos recogidos en los hospitales encuestados se deben al perfil de servicio , la ocupación, la ociosidad, a menudo elevado, el valor de la compra de materiales y la medicina, y especialmente a los costes de personal. Determinar el coste por proceso es útil para establecer el precio promedio de cada procedimiento médico , estimar los costos de los paquetes de negociación ; determinar el margen bruto de los procedimientos del hospital y los márgenes brutos correspondientes a las especialidades médicas ; estimar la rentabilidad de los procedimientos médicos hospitalarios y especialidades médicas, y confrontar el valor de factura de los gastos de procedimiento médico hospitalario.

\section{INTRODUÇÃO}

A incorporação tecnológica, a negociação por protocolos médicos (pacotes), novos medicamentos, concorrência entre os tomadores de serviços tornam a mensuração de custos fundamental para a sobrevivência de uma instituição hospitalar (CARNIELO, 2011).

Aliado a isto, o aumento da expectativa de vida da população, obrigou os serviços a atenderem um maior contingente de pacientes com mais de 60 anos, onde os agravos são mais frequentes, complicados e onerosos, e as doenças crônico-degenerativas, que aumentaram proporcionalmente às doenças infecto-contagiosas, exigindo internações, equipamentos, exames e tratamentos sofisticados.

Assim, as técnicas e materiais utilizados na prática da medicina têm sido aprimorados e sofisticados continuamente e sua incorporação nas ações rotineiras de assistência médica passou a exigir mão-de-obra mais especializada e aquisição de novos equipamentos, que têm maiores custos operacionais e de manutenção.

Inseridos neste contexto, os hospitais representam quase a metade das despesas totais consagradas ao setor de saúde tornando-se empresas altamente complexas, o que implica na utilização de instrumentos econômicos, gerenciais e financeiros para sua administração possibilitando assim um melhor controle dos gastos e do custo da atenção médico-hospitalar com ganhos de eficiência e efetividade, melhoria na qualidade dos serviços oferecidos, na equidade e na satisfação dos clientes (ALVES, 1997).

Segundo Bruger (1997) a indústria de cuidados à saúde cresceu muito e se tornou uma das maiores indústrias da nação, não somente nos Estados Unidos, mas também em outros países. Porém, todos os interessados, os governos federal, estadual e local, as financiadoras e mesmo alguns grupos de consumidores estão especialmente preocupados com o aumento crescente dos gastos dedicados à saúde e o seu aparente baixo nível de eficácia.

Os recursos destinados à saúde são poucos sendo que, os gastos com programas de combate à fome, saneamento básico e programas especiais, que antes não faziam parte do orçamento da saúde, agora estão nele inclusos.

Assim, o contexto do setor saúde no Brasil torna a gestão de custos hospitalar uma ferramenta imprescindível. Porém, reproduzindo o questionamento de Carnielo (2011, p. 34) "quantas organizações hospitalares brasileiras realmente sabem quanto custa os seus procedimentos médicos realizados, ou em outras palavras, o seu produto?" Segundo ainda o referido autor, "normalmente os hospitais que possuem gestão de custos, limitam-se aos custos departamentalizados, que isoladamente, permite reduzidas decisões gerenciais" (CARNIELO, 2011, p. 34).

Controlar e reduzir custos, formar o preço de venda dos serviços e arquitetar a estrutura operacional, entende-se são extremamente necessário para o bom funcionamento de qualquer organização, inclusive o hospital. 
Segundo a Federação Brasileira de Hospitais, desde 1999 até 2005 pelo menos 30 hospitais privados fecharam as portas (NEVES, 2003 apud OLIVEIRA et al., 2007), sendo que muitos destes hospitais encerraram suas atividades devido a falhas no processo de gerenciamento de custos.

Portanto, o registro e a gestão estratégica de custos hospitalares são necessários nas instituições hospitalares, visto que enquanto o primeiro serve de instrumento eficaz de gerencia e acompanhamento dos serviços, o segundo permite a implantação de medidas corretivas que visem a um melhor desempenho das unidades, com base na possível redefinição das prioridades essenciais, aumento da produtividade e racionalização do uso de recursos dentre outras medidas administrativas (ABBAS, 2001).

Assim, segundo Carnielo (2011, p. 34):

\begin{abstract}
a busca por um modelo de custos que apóie a tomada de decisão deve ser uma das principais preocupações dos hospitais. Mas especificamente, o modelo deve ser capaz de fornecer o levantamento de custos por especialidades mais rentáveis, os resultados por convênios, por planos, por médicos e por procedimentos. Entretanto, devem-se tomar alguns cuidados importantes e relevantes: a ampla discussão entre os colaboradores e gestores sobre a metodologia adotada, a mobilização das pessoas, a qualidade e validação dos dados e, a mais importante, a abordagem sistêmica. $\mathrm{Na}$ implantação de um modelo de gestão de custos entender os processos, a rede de atividades e a dinâmica hospitalar são variáveis fundamentais que determinam o sucesso ou não da ferramenta de custeio.
\end{abstract}

Diante da importância dos hospitais conhecerem seus custos, o objetivo deste trabalho é apresentar o cálculo dos custos do procedimento médico hospitalar parto cesárea em seis hospitais brasileiros que utilizam o método de custeio das seções homogêneas.

Para tanto, este artigo está dividido, além desta introdução, em mais quatro seções. A segunda seção apresenta a revisão literatura onde, inicialmente, discorre sobre o que é um hospital e sua função. Em seguida apresenta o método de custeio RKW, ou método das seções homogêneas, explicando sua lógica de funcionamento e como obter o custo de um procedimento médico hospitalar. Na terceira seção apresenta a metodologia da pesquisa e na quarta seção expõe apresenta o cálculo do custo do procedimento médico hospitalar parto cesárea em seis hospitais brasileiros. Por fim, a quinta e última seção apresenta as considerações finais do estudo.

\title{
2 REVISÃO BIBLIOGRÁFICA
}

\subsection{OS HOSPITAIS: CONCEITO E CARACTERÍSTICAS}

Para Almeida (1983, p.205), precursor dos estudos sobre administração hospitalar no Brasil, hospital é:

uma instituição destinada ao diagnóstico e tratamento de doentes internos e externos; planejada e construída ou modernizada com orientação técnica; bem organizada e convenientemente administrada consoante padrões e normas estabelecidas, oficial ou particular, com finalidades diversas; grande ou pequena; custosa ou modesta para atender os ricos, os menos afortunados, os indigentes e necessitados, recebendo doentes gratuitos ou contribuintes; servindo ao mesmo tempo para prevenir contra a doença e promover a saúde, a prática, a pesquisa e o ensino da medicina e da cirurgia, da enfermagem e da dietética, e das demais especialidades afins.

Em outras palavras, um hospital é uma unidade econômica que possui vida própria e, 
difere das outras empresas porque o seu objetivo ou "produto" básico é a manutenção ou restabelecimento da saúde do paciente.

Kotler, citado por Esteves (1992, p.48), define como produto: "Qualquer coisa que possa ser oferecida a um mercado para satisfazer uma necessidade. Inclui a aquisição ou consumo de objetos físicos, serviços, pessoas, lugares, organização e idéias....".

Logo, em uma organização hospitalar, um produto hospitalar é uma série de serviços prestados a um paciente como parte do processo de tratamento, controlado pelo médico.

Médici e Marques (1996, p.49) destacam que as instituições hospitalares possuem peculiaridades que são perfeitamente compreendidas quando "o indivíduo, ao procurar a assistência médica, não o faz por livre vontade, mas sim por necessidade, isto é, por circunstâncias alheias ao seu desejo. Ao ingressar num serviço de saúde, o indivíduo não sabe que tipo de intervenção irá sofrer ou que tipo exame ou medicamento irá consumir".

As organizações hospitalares diferem de outros tipos de organizações, pois suas atividades possuem características próprias, conforme cita Machline et al. (1983):

- serviços de atenção e tratamento personalizado a pacientes individuais;

- dependência das necessidades e demanda de seus clientes;

- definição das responsabilidades dos diferentes membros e pouca tolerância a erros;

- trabalho diversificado e com pouca padronização em que pessoas cuidam de pessoas, participando ativamente do processo de produção;

- pouco controle sobre seus trabalhadores (principalmente os médicos) e sobre os pacientes;

- organização não baseada em uma linha única de autoridade; já que o administrador hospitalar detém menos autoridade e poder relação a outras organizações;

- organização formal, até certo ponto burocrática e autoritária, cuja operacionalidade repousa no arranjo do trabalho convencionalmente hierarquizado e em regras rígidas e impessoais. É, porém, uma organização altamente especializada, departamentalizada e profissionalizada que não pode funcionar efetivamente sem uma coordenação interna, motivação, autodisciplina e ajustes informais e voluntários de seus membros.

Assim, segundo Porter (2007) prestar serviços de saúde em um hospital, é algo complexo que envolve miríades de atividades e desafios e os prestadores precisam lidar com contratos com planos de saúde, negociações de pagamento, cumprimento de exigências regulamentares, assimilação de novas tecnologias médicas, melhoria do atendimento a clientes e recrutamento e retenção de pessoal.

Portanto, a gestão de custos em hospitais é um importante instrumento de gerenciamento e controle dos custos incorridos.

A literatura apresenta vários métodos de custeio, entre eles o custeio por absorção, método das seções homogêneas e o custeio baseado em atividades são alguns exemplos. Todos estes métodos apresentam vantagens e desvantagens.

Porém, cita Matos (2002), que devido a complexidade deste tipo de organização, pelas informações e pelos controles gerados, o método das seções homogêneas é o mais viável de ser implantado em um hospital.

\subsection{MÉTODO DAS SEÇÕES HOMOGÊNEAS - RKW E CUSTO POR PROCEDIMENTO}

O método das seções homogêneas, segundo Bornia (2010), teve sua origem na Europa, no início do século XX. Hoje, segundo o referido autor, é o mais utilizado no Brasil e no mundo.

Este método é composto de três etapas. Na primeira etapa, divide-se a empresa em 
centros de custos, que segundo Selig (1993) são as unidades que realizam atividades homogêneas dentro do processo produtivo da organização. Pode-se entender por homogeneidade: "Um agrupamento real ou ideal de meios materiais e humanos agindo com a mesma finalidade, usando os mesmos meios, participando das mesmas despesas e possuindo, pois, aproximadamente as mesmas características" (SELIG, 1993, p.47).

Tendo sido definidos os centros de custos, distribuem-se todos os itens de custos e despesas a nestes centros, através de bases de rateio, obtendo-se os custos totais para cada centro de custos.

$\mathrm{Na}$ segunda etapa, os centros de custos que são os responsáveis pelas atividades de caráter geral ou comum do hospital (administração, departamento pessoal, diretoria, contabilidade, faturamento, almoxarifado e compras, dentre outros) e os centros de custos intermediários que correspondem às atividades complementares ou de apoio da unidade hospitalar (farmácia, lavanderia e rouparia, central de esterilização, higiene e limpeza, recepção, governança e, dentre outros) repassam seus custos entre eles e para os centros de custos finais (centro cirúrgico, unidade de internação, ambulatório, tomografia, radiologia e pronto-socorro, dentre outros), ou seja, através do rateio sequencial ou gradativo (step down) cada centro de custos transfere seu custo total à todos os centros de custos que tenham prestado serviços, ou seja, àqueles que sucedam no plano hierarquizado de centros de custo ("efeito cascata").

Segundo Mattos (1998), permite assim, a transferência de custo também entre centros de custo do mesmo grupo. Em contrapartida, exige um grande rigor na hierarquização dos centros alocados em cada um dos grupos, de forma que, no processo de interação de troca de serviços, tenha prioridade, no rateio de seus custos para os demais, aqueles cujos serviços prestados tenham maior relevância econômica do que os recebidos. Os centros de custos que já foram rateados, não receberão rateios dos demais centros, evitando-se assim, a formação de resíduos de custos não apropriáveis aos produtos.

A figura 1 apresenta a matriz de custos, onde na primeira coluna listam-se os custos e despesas do período para em seguida, nas colunas 2 e 3 , listarem-se o valor destes itens e as bases de rateio definidas. As colunas seguintes correspondem aos centros de custos.

\begin{tabular}{|l|l|l|l|l|l|}
\hline $\begin{array}{c}\text { Itens de Custos / } \\
\text { Despesas }\end{array}$ & Valor & $\begin{array}{c}\text { Bases de } \\
\text { Rateio }\end{array}$ & $\begin{array}{c}\text { Centros } \\
\text { Administrativos }\end{array}$ & $\begin{array}{c}\text { Centros } \\
\text { Auxiliares }\end{array}$ & $\begin{array}{c}\text { Centros } \\
\text { Produtivos }\end{array}$ \\
\hline & & & & \\
& & & & \\
\hline & & & \\
\end{tabular}

Figura 1 - Matriz de custos

Fonte: Adaptado de Bornia (2010)

Por fim, na terceira etapa, os custos são alocados dos centros finais aos produtos.

Cita ainda o referido autor que a segmentação mais usual aplicada a um hospital compreende os conceitos de centros de custos produtivos, centros de custos auxiliares e administrativos (MATOS, 2002). O quadro 1 apresenta este agrupamento de centros de custos para hospitais em geral: 


\begin{tabular}{|l|}
\hline CENTROS ADMINISTRATIVOS \\
\hline -- Departamento Pessoal \\
-- Diretoria \\
-- Serviço de Arquivo Médico e Estatística \\
-- Contabilidade \\
-- Faturamento \\
-- Almoxarifado \\
-- Compras \\
\hline CENTROS AUXILIARES \\
\hline -- Centro de Esterilização \\
-- Lavanderia e Rouparia \\
-- Nutrição e Dietética \\
\hline -- Farmácia \\
-- Higienização e Limpeza \\
-- Recepção da Internação \\
-- Recepção do Pronto Socorro \\
-- Governança \\
-- Manutenção \\
\hline CENTROS PRODUTIVOS \\
\hline -- Ambulatório \\
-- Pronto Socorro \\
-- Ultrassonogradia \\
-- Tomografia \\
-- Radiologia \\
-- Laboratório de Análises Clínicas \\
-- Centro Cirúrgico \\
-- Unidade de Tratamento Intensivo \\
-- Unidade de Internação \\
\hline
\end{tabular}

Quadro 1 - Centros de custos

Fonte: Elaborado pelos autores

Assim, o método das seções homogêneas favorece o controle analítico dos custos por responsabilidade (centros de custos) além de comparação de custos com receitas, custos unitários, tabelas de preços, custos incorridos e volumes de produção.

Segundo Matos (2002, p. 97-98):

a apropriação de custos por centros de custos e o custeio por procedimentos hospitalares representam as duas formas mais usuais de expressão do custo de um serviço gerado por uma empresa hospitalar. O custo gerado pela apropriação dos centros de custos corresponde às unidades de serviços produzidas em cada um dos centros de custos definidos para o hospital. As expressões de custo unitário associadas a cada um dos centros de custos corresponderão, portanto, a uma diária hospitalar, a uma taxa de sala cirúrgica, a uma consulta, a um exame, entre outros. $\mathrm{O}$ custo dos procedimentos hospitalares corresponde a uma sequência de cálculos, os quais compreenderão os custos unitários gerados pelos centros de custos combinados com a intensidade dos referidos insumos.

O custo por procedimento citado por Matos é o somatório dos itens incorridos no processo de produção mais a utilização dos referidos insumos (materiais e medicamentos) consumidos no tratamento dos pacientes. Pode-se dizer, em outras palavras, que o custo do procedimento é o custo do paciente, ou seja, todo o consumo de insumos é registrado na conta 
do paciente e a respectiva conta refere-se a pessoa (paciente) e a determinado procedimento (que é o que levou o paciente a ser atendido no hospital). Ressalta-se que em uma conta podem ser acumulados vários procedimentos.

Com isso, fica evidente que o método das seções homogêneas fornece os custos dos centros de custos, ou seja, o custo de uma diária hospitalar, de uma taxa de sala cirúrgica, de uma consulta, de um exame, entre outros. E, com esta informação é possível avançar e obter o custo por procedimento, ou seja, considera-se o custo do serviço prestado pelo hospital (custos dos centros de custos produtivos) mais o consumo de materiais e medicamentos que o paciente utilizou.

\section{METODOLOGIA}

Empregando a classificação proposta por Silva e Menezes (2001), sob o ponto de vista da sua natureza é pesquisa aplicada, pois objetiva gerar conhecimentos para aplicação prática, dirigidos à solução de problemas específicos, ou seja, objetiva apresenta a importância da obtenção dos custos por procedimentos em hospitais.

Quanto à forma de abordagem do problema a pesquisa é quantitativa qualitativa, pois traduz em número os procedimentos realizados em hospitais e, também, incorre em interpretações dos custos obtidos nos seis hospitais pesquisados.

Quanto aos objetivos é pesquisa exploratória, pois proporciona uma visão geral do assunto investigado buscando aprofundar os conhecimentos sobre ele. Também é pesquisa explicativa, pois descreve e explica as características dos de determinada população.

Quanto aos procedimentos técnicos, é pesquisa bibliográfica quando elaborada a partir de livros, artigos científicos, dissertações e teses, ou seja, elaborada através da contribuição de outros autores. É também pesquisa documental, pois as informações utilizadas foram reelaboradas de acordo com os objetivos do artigo. E, por fim, também pode ser caracterizada como levantamento, pois, segundo Beuren (2003, p. 86), "levanta informações que podem ser úteis para estudos futuros mais específicos ou mesmo mapear a realidade de determinada população ou amostra". Neste caso, foi feita uma pesquisa nos hospitais com o objetivo de conhecer o comportamento em relação aos seus custos por procedimentos.

\section{RESULTADOS DA PESQUISA}

Foram analisados seis hospitais de médio porte sendo um no Rio de Janeiro, dois em Campo Grande, um em São Paulo, um em Belém e um em Manaus. As informações coletadas referem-se ao período de janeiro a outubro do ano de 2010. Para preservar a identidade dos hospitais pesquisados, estes serão aqui denominados de Hospital A, B, C, D, E e F.

Todos os hospitais são filantrópicos e possuem o método das seções homogêneas implantado o que possibilita os custos dos centros produtivos. O custo médio da unidade de internação, do ambulatório, do pronto-socorro, da ultrassonografia, da tomografia, da radiologia, do laboratório de análises clínicas, do centro cirúrgico e da unidade de tratamento intensivo é obtido através da divisão do custo total do centro de custos (exclui-se destes, os custos com materiais e medicamentos e, também, o custo com honorários médicos, visto que, o médico recebe pelos serviços prestados diretamente do convênio médico), pelo número de unidades produzidas pelo centro de custos.

A conta do paciente recebe o custo médio unitário dos centros de custos que prestaram serviços a estes e os materiais e medicamentos são fornecidos pelo almoxarifado e pela farmácia.

O paciente ou usuário do hospital é o repositório de todos os serviços gerados pela 
empresa. Assim, através da conta do paciente, é possível obter o custo por procedimento realizado. A figura 2 apresenta o custo por procedimento obtido nos hospitais pesquisados.

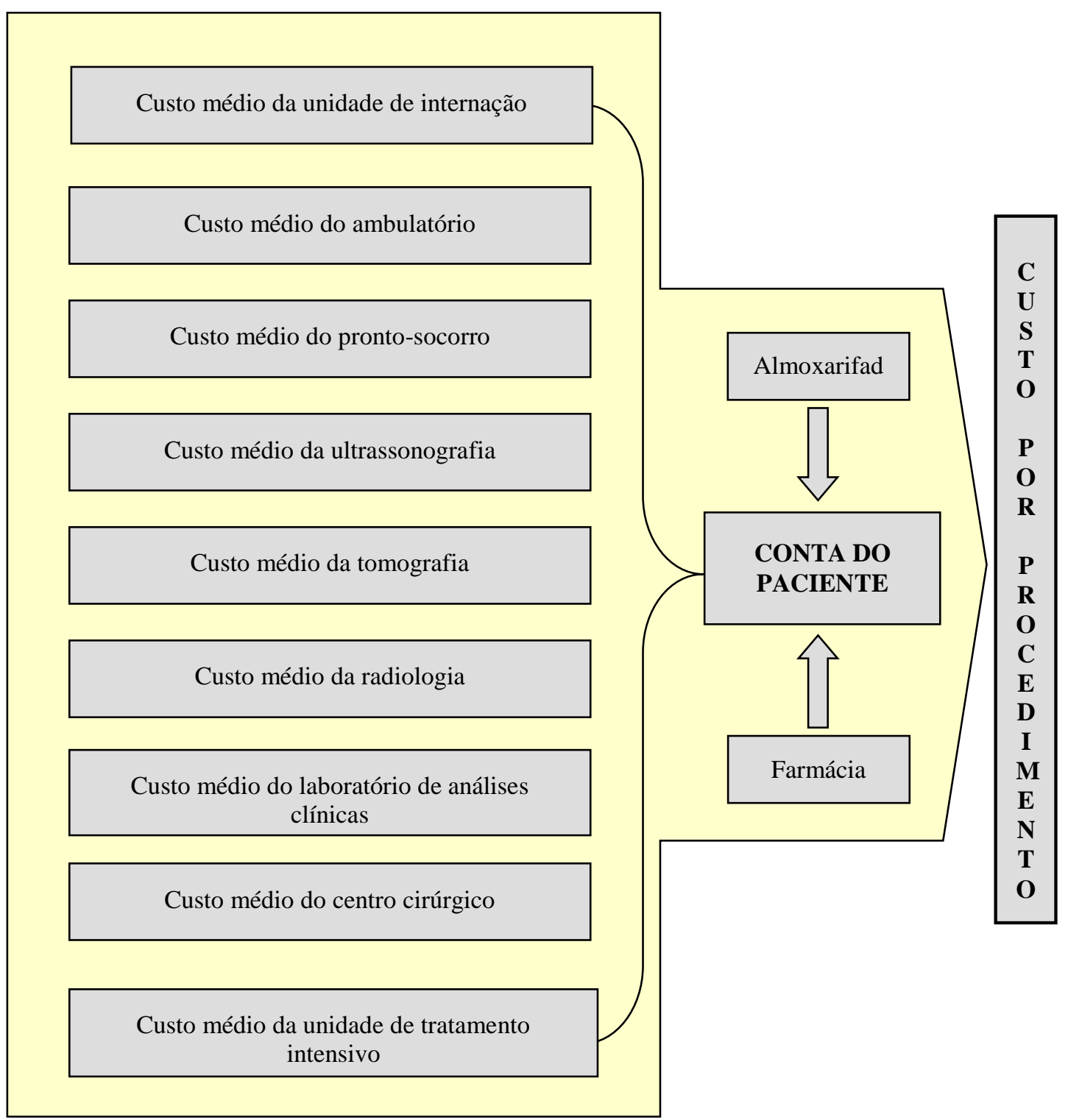

Figura 2 - Custo por procedimento

Fonte: Elaborado pelos autores

A tabela 1 apresenta o cálculo do custo do procedimento parto cesárea nos seis hospitais selecionados. A escolha deste procedimento se deve a representativa incidência nos seis hospitais escolhidos. Foi feita uma coleta de dados (amostragem) de dez contas de pacientes em cada hospital no período de janeiro a outubro de 2010.

$\mathrm{Na}$ tabela, a segunda coluna corresponde ao custo médio dos centros de custos produtivos pelos quais a paciente que fez parto cesárea passou. Este custo médio foi obtido através do método das seções homogêneas.

A terceira coluna corresponde aos custos de materiais e medicamentos fornecidos pelo almoxarifado e pela farmácia, conforme prescrição médica. O critério de avaliação destes itens é a média ponderada móvel, que considera o custo dos materiais e medicamentos pelo seu valor médio, obtido através da divisão do valor total do estoque pela quantidade de unidades existentes em estoque. 
Assim, o somatório da segunda e da terceira coluna, ou seja, do custo dos centros produtivos mais os custos dos materiais e medicamentos, resulta no custo do procedimento parto cesárea para os hospitais.

Tabela 1 - Apuração do custo do procedimento médico - parto cesárea

\begin{tabular}{lrrr}
\hline HOSPITAL & $\begin{array}{c}\text { CUSTO DOS } \\
\text { CENTROS } \\
\text { PRODUTIVOS }\end{array}$ & $\begin{array}{c}\text { ALMOXARIFADO/ } \\
\text { FARMÁCIA }\end{array}$ & $\begin{array}{c}\text { CUSTO DO } \\
\text { PROCEDIMENTO }\end{array}$ \\
\hline Hospital A & $1.884,51$ & 190,87 & $2.075,38$ \\
Hospital B & $2.031,18$ & 539,39 & $2.570,57$ \\
Hospital C & 700,50 & 159,22 & 859,72 \\
Hospital D & 659,30 & 139,39 & 798,69 \\
Hospital E & $1.483,48$ & 224,27 & $1.707,76$ \\
Hospital F & $1.622,67$ & 295,21 & $1.917,88$ \\
MÉDIA & $1.396,94$ & 258,06 & $1.655,00$ \\
\hline
\end{tabular}

Fonte: Elaborado pelos autores

As variações nos dados apurados se devem as particularidades da cada hospital, ou seja, ao perfil de atendimento, a taxa de ocupação, a ociosidade, muitas vezes elevada, ao valor da compra dos materiais e medicamento e, principalmente, ao custo com pessoal, considerado o principal item de custos de um hospital, ou seja, um dos custos mais elevados.

\section{CONSIDERAÇÕES FINAIS}

O setor de saúde passa por um momento em que é necessário lidar com os avanços das pesquisas, tecnologias inovadoras de diagnóstico, aumento da idade média da população, contratos, exigências de órgãos regulamentadores, negociações de pagamentos, constante busca pela qualidade, eficiência no tratamento e a gestão de pessoas. Assim, a gestão de custos é uma ferramenta imprescindível para a sobrevivência frente à competitividade do setor, onde quem determina os preços é o mercado, e a única maneira de sobreviver é gerenciando os custos.

A determinação do custo por procedimento (que pode ser subdividido em: por médico, por especialidade e por convênio) é importante, porque afetará um grande número de ações a serem tomadas no hospital.

Uma correta determinação do custo do procedimento servirá de parâmetro para traçar as estratégias de gestão de custos: estabelecer o preço médio de cada procedimento médico; estimar os custos de negociação de pacotes; determinar as margens brutas associadas às especialidades e procedimentos médicos; estimar a rentabilidade das especialidades e procedimentos médicos; e confrontar com o valor faturado o custo do procedimento médico.

Porém, para obter o custo do procedimento é necessário que o hospital tenha um método de custos implantado. $\mathrm{O}$ método das seções homogêneas é o mais adequado para as características e complexidade de um hospital. Ressalta-se porém, que para uma apuração de custos precisa faz-se é necessário, também, que todos os setores envolvidos no processo de apuração enviem as informações pertinentes e confiáveis para o setor de custos.

O custo dos serviços, apurado pelo método das seções homogêneas, proporciona os dados necessários para que, com a junção dos demais dados lançados na conta do paciente (custos diretos - material e medicamento - e outros lançamentos feitos pelo faturamento), seja 
possível inferir na apuração do custo por procedimento, que é o objetivo final do ciclo da apropriação dos custos, uma vez que o hospital é remunerado pelos procedimentos.

Assim, o custo por procedimento médico hospitalar fornece a informação estratégica para a tomada de decisão.

O objetivo geral do trabalho - apresentar o cálculo dos custos do procedimento médico hospitalar parto cesárea em seis hospitais brasileiros - foi alcançado. O item 4, apresenta como incorrer no cálculo do custo do procedimento médico hospitalar parto cesárea em seis hospitais brasileiros.

Para estudos futuros, sugere-se que, com base nos custos do procedimento parto cesárea obtidos, sejam feitas análises para verificar se os custos estão adequados com a remuneração que o hospital recebe pelo procedimento e se há possibilidade de melhorias, dentre elas, a padronização, a negociação de preços e o aprimoramento da gestão dos custos fixos.

\section{REFERÊNCIAS}

ABBAS, Katia. Gestão de custos em organizações hospitalares. Dissertação (Mestrado em Engenharia de Produção) - Programa de Pós-Graduação em Engenharia de Produção, Universidade Federal de Santa Catarina, Florianópolis, 2001.

ALMEIDA, José Jesus de. Sistemas de custos em organizações hospitalares - estudo de casos. Dissertação (Mestrado em Ciências Contábeis) - Instituto Superior de Estudos Contábeis da Fundação Getúlio Vargas, Fundação Getúlio Vargas, Rio de Janeiro, 1987.

ALVES, A. Financiamento e eficiência em dois hsopitais provados filantrópicos paulistas. Tese (Doutorado em Saúde Pública) - Faculdade de Saúde Pública, Universidade de Saúde Pública, São Paulo, 1997.

BEUREN, Ilse Maria. Como elaborar trabalhos monográficos em contabilidade: teoria e prática. In: LONGARAY, André Andrade; RAUPP, Fabiano Maury; SOUSA, Marco Aurélio Batista; COLAUTO, Romualdo Douglas; PORTON; Rosimere Alves de Bona; BEUREN, Ilse Maria (Org.). São Paulo: Atlas, 2003.

BORNIA, Antonio Cezar. Análise gerencial de custos: aplicação em empresas modernas. 3. Ed. São Paulo: Atlas, 2010.

BRUGER, Newman, et al. Financial management: concepts and applications for health care organizations. Dubuque, Iowa: Kendall/Hunt, 1997.

CARNIELO, Marcelo Tadeu. Gestão de custos: pensando questões relevantes na definição de custos hospitalares. Gestão e Tecnologia hospitalar, v. 5, p. 34-36, fev. 2011. Disponível em: <www. gthospitalar.com.br/files/editions/ed1298479725.htm>. Acesso em: 05/05/2011.

ESTEVES, Maria Josefa Vilar. A utilização de custos em hospitais paulistas: um estudo preliminar. Dissertação (Mestrado em Saúde Pública) - Faculdade de Saúde Pública, Universidade de São Paulo, São Paulo, 1992.

LEONCINE, Maury. Sistemática para apuração de custos por procedimento médico hospitalar. Dissertação (Mestrado em Engenharia de Produção) - Programa de PósGraduação em Engenharia de Produção, Universidade Federal de Santa Catarina, Florianópolis, 2010. 
MACHLINE, C. et al. O hospital e a visão administrativa contemporânea. São Paulo: Pioneira, 1983.

MATOS, Afonso José de. Gestão de custos hospitalares: técnicas, análise e tomada de decisão. São Paulo: Editora STS, 2002.

MATTOS, José Geraldo de. Sistema de apropriação de custos do hospital universitário da UFSC: um diagnóstico da situação atual. Monografia (Especialização em Gestão Hospitalar) - Universidade Federal de Santa Catarina, Florianópolis, 1998.

MEDICI, André Cézar; MARQUES, Rosa Maria. Sistemas de custo como instrumento de eficiência e qualidade dos serviços de saúde. Cadernos Fundap. Rio de Janeiro: FGV, p.4759, jan./abr. 1996.

OLIVEIRA, Alexandre David da Silva; BRUNI, Adriano Leal; PAIXÃO, Roberto Brazileiro; CARVALHO JÚNIOR, Cesar Valentim. Estratégia, custos e hospitais: um diagnóstico na cidade de Salvador, Bahia (2006). XIV Congresso Brasileiro de Custos. Anais eletrônicos... João Pessoa - PB, 2007.

PORTER, Michael E. Repensando a saúde: estratégias para melhorar a qualidade e reduzir custos / Michael E. Porter, Elizabeth Olmsted; tradução de Cristina Bazan. Porto Alegre: Bookman, 2007.

SELIG, Paulo Maurício. Gerência e avaliação do valor agregado empresarial. Tese (Doutorado em Engenharia de Produção) - Programa de Pós-Graduação em Engenharia de Produção, Universidade Federal de Santa Catarina, Florianópolis, 1993.

SILVA, Edna Lúcia da. MENEZES; Estera Muszkat. Metodologia da pesquisa e elaboração de dissertação. 3. ed. Florianópolis: UFSC/PPGEP/LED, 2007. 